\title{
Social circumstances and dietary intake
}

While the debate over unhealthy food environments and personal responsibility continues ${ }^{1}$, the basic concept that social circumstances affect dietary intake is generally wellaccepted. How social factors exert their effects and whether the processes are amenable to change, however, are issues of primary interest and importance to public health nutrition professionals. In this edition of Public Health Nutrition, two articles examine social circumstance and diet in novel ways.

Mishra et $a l^{2}$ examine the associations of childhood social class and region of residence with dietary patterns at age 43 years using data from the Medical Research Council's 1946 British Birth Cohort. The research has two unique aspects: the availability of longitudinal data to describe changes in social class and region of residence from age 4 to age 43 , and the use of principal components analysis to describe patterns of food intake in the cohort. The result is a fascinating picture of how social and regional mobility (or stability) affect what people eat as middle-aged adults. Among their findings is that individuals who moved from manual classes in childhood to nonmanual classes at age 43 appeared to adopt a diet befitting the education and income levels of the non-manual classes - namely, higher consumption of the 'healthaware' (high-fibre breakfast cereals, whole-grain breads, fruits) and 'dinner party' (coffee, white wine, cream) patterns. Regional migration was also associated with dietary changes, although region-associated dietary patterns appeared to be, as the authors state, 'more resilient to change' than were social class-related patterns.

Kim and Sobal $^{3}$ examine another aspect of social circumstance and diet in their investigation of religion and religiosity. The premise of their work is that religion might affect dietary behaviours by defining norms and values that distinguish their religious group from others, and by serving as a source of social support and networks that help develop and maintain healthful behaviours. They find some difference in fat intake by religious denomination among women, but no effect for different aspects of religiosity, such as religious attendance or religious commitment. In offering other possibilities for how religion might influence dietary behaviour, they suggest an additional insight - that, rather than encourage healthier dietary habits, religion may simply support the cultural food norms of the local region.

That regional food norms emerge as an underlying and apparently less tractable determinant of dietary intake in both studies is perhaps not surprising. Residents of a local region likely share the same 'cultural rules for patterning food intake ${ }^{4}$ - that is, share the same understanding about which foods can be combined into recognisable dishes and organised into meals. Such cultural rules provide the template for what people choose to consume and may be less amenable to change. Other segments within a culture, however, defined by social class or religion for example, may be more useful as agents of change. Thus, a rise in social class may bring with it better education and higher income, and with that, better diet, as Mishra et al. confirm with their 'health-aware' dietary pattern. Or participation in religious activities may facilitate access to social networks and resources that can serve as vehicles for nutrition education programmes 5 .

Kim and Sobal cite Link and Phelan ${ }^{6}$ in arguing for the importance of 'contextualising' proximate risk factors such as poor dietary intake - to understand how healthrelated behaviours are developed and maintained. As Link and Phelan state,

Understand[ingl how people come to be exposed to individually-based risk factors such as poor diet [...] is important so that we can design more effective interventions [...] [E]fforts to reduce risk by changing behavior may be hopelessly ineffective if there is no clear understanding of the process that leads to exposure.

Attention to upstream determinants of dietary behaviours and disease is a distinguishing feature of many of the articles selected for publication in Public Health Nutrition. The effects of social, cultural, political and economic circumstances on diet are a crucial aspect of our field of research and practice. What is needed to advance the field further are studies with innovative designs and methods that offer new perspectives on which social circumstances are important, and that illustrate clearly and convincingly how they affect diet. Continued work in this area serves as a reminder that interventions to improve diet and health must occur at many levels and not just target individual behaviours.

Marilyn Tseng

Editor

\section{References}

1 Zernike K. Food fight: is obesity the responsibility of the body politic? Week in Review. The New York Times, 9 November 2003; 3 .

2 Mishra GD, Prynne CJ, Paul AA, Greenberg DC, Bolton-Smith C. The impact of inter-generational social and regional circumstances on dietary intake patterns of British adults: 
results from the 1946 British Birth Cohort. Public Health Nutrition 2004; 7(6): 737-44.

$3 \mathrm{Kim} \mathrm{KH}$, Sobal J. Religion, social support, fat intake and physical activity. Public Health Nutrition 2004; 7(6): 773-81.

4 Goode JG. Cultural patterning and group-shared rules in the study of food intake. In: Messer E, ed. Research Methods in Nutritional Anthropology. Tokyo: United Nations University Press, 1989.
5 Resnicow K, Jackson A, Braithwaite R, DiIorio C, Blisset D, Rahotep S, et al. Healthy Body/Healthy Spirit: a church-based nutrition and physical activity intervention. Health Education Research 2002; 17: 562-73.

6 Link BG, Phelan J. Social conditions as fundamental causes of disease. Journal of Health and Social Behavior 1995; 36: 80-94. 